\title{
Multispectral Image Compression for various band images with High Resolution Improved DWT SPIHT
}

\author{
V. Bhagya Raju ${ }^{1}$, Dr. K. Jaya Sankar ${ }^{2}$, Dr. C. D. Naidu ${ }^{3}$ and Srinivas Bachu ${ }^{4}$ \\ ${ }^{1}$ Research Scholar, ${ }^{2}$ Principal, ${ }^{3}$ Principal and ${ }^{4}$ Assistant Professor \\ ${ }^{1}$ JNTU Hyd, ${ }^{2}$ Vasavi College of Engineering, ${ }^{3}$ VNR VJIET and ${ }^{4}$ GNITC, TS, India \\ ${ }^{1}$ vbhagya01@gmail.com, ${ }^{2}$ kottareddyjs@gmail.com, ${ }^{3}$ principal@vnrvjiet.in \\ ${ }^{4}$ bachusrinivas@gmail.com
}

\begin{abstract}
Satellite imageries which comprises of various multispectral spectral bands pertaining to spectral and spatial information of the images acquired by latest multispectral sensor technology are rapidly increasing day by day in the recent years for onboard satellite remote sensing applications. A lossy multispectral image compression is desired by the exploitation of the redundancies present in the spatial and spectral information while preserving the vital and crucial information of the image objects to a certain extent. In this paper a novel approach is proposed for lossy multispectral image compression which is an extension to the earlier existing algorithms. In this proposed method the multispectral images are first enhanced with interpolation based super resolution technique to estimate a hi-resolution (HR) image from a low-resolution (LR) input image. Secondly the decorrelated spectral bands transformed by discrete wavelet transform $(D W T)$, which contain maximum entropy, are selected and these representative spectral bands are quantized and encoded using Improved SPIHT (ISPIHT) algorithm. The algorithm has been designed for the optimization of maximum coding efficiency and for high compression ratio of bits per pixel per band when compared with the well known compression techniques.
\end{abstract}

Keywords: Multispectral Images, DWT, ISPIHT, LIBT, LIST

\section{Introduction}

Satellite borne spectrometers which cover large tracts of earth's surface contain large amount of information over visible bands contained in the multiple bands of multispectral images in the Electromagnetic (EM) spectrum when compared to RGB color images. Multispectral cameras are more expensive due to their high resolution and acquisition capabilities when compared to their RGB counterparts. Multispectral cameras which contain separate sensor arrays for each band of the EM spectrum requires large number of optical and mechanical parts for image acquisition thereby increasing the cost and size of the cameras. In remote sensing applications storage and transmission of multispectral images introduces difficulties due to large amount of data present in these images. In order to mitigate these problems there is a need to reduce the data size by means of multispectral image compression. This is achieved by a high performance and a low complexity compression codec like ISPIHT. The traditional methods of multispectral image compression are mainly based on dimensionally reduction through principal component analysis (PCA) [1] [2] [3] or through direct vector quantization and differential pulse code modulation (DPCM). Energy compaction of the decorellated spectral bands is the main advantage of the PCA method whereas the data dependency for the calculation of the covariance matrix among decorellated frequency bands is the main disadvantage. The correlation between multispectral bands is expressed through, Karhunen-Loeve transform (KLT) and Vector quantization (VQ) which is often used in 
compression [4]. KLT is normally used for a few number of spectral bands but computing KLT for 224 bands is usually not practical for AVIRIS multispectral images. Embedded block coding with optimized truncation (EBCOT) scheme is implemented for JPEG 2000 coder and decoder with wavelet based compression method. Discrete cosine transforms (DCT) is not so impressive due its poor image quality in spite of many advantages like approximating the signal to large extent. Recently, an adaptation of the context based adaptive lossless image codec (CALIC) to on board multispectral data compression has been presented in [5]. Mean-normalized vector quantization (M-NVQ) is proposed by Ryan and Arnold for lossless AVIRIS compression [6]. So looking back to these problems a novel approach has been proposed for lossy compression of high resolution multispectral images using DWT and ISPIHT. We investigate various lossy compression algorithms for performance evaluation of multispectral images and compare them with the proposed method. The compared algorithms include DCT KLT [7], DWT SPIHT [2] algorithms respectively.

The need for multispectral image compression is explained in section 2. A high resolution image obtained from a low resolution image using interpolation based super resolution technique and encoding the maximum entropy decorrelated spectral bands using DWT IPIHT is discussed in section 3. The performance evaluation is discussed in section 4.The results obtained from various bands of multispectral images are compared to the performance of DCT KLT, DWT SPIHT and proposed DWT Improved SPIHT in terms of PSNR, CR, BER, MSE and SSIM in section 5. In section 6 we conclude and present perspectives for future applications.

\section{Need for Compression}

The demand for image compression for image storage and transmission bandwidth is rapidly increasing in spite of mass storage devices, high processor speed devices for digital communication systems. A simple uncompressed geographic information system (GIS) multispectral image like world map ( $3 \times 2 \frac{1}{2}$ feet in size, for example) requires 1 GB storage. To retrieve this data remotely over internet with an internet transmission speed of 1 Mbps (bits per second), requires more than 2 hours. Thus there is a need to compress these images by exploiting their redundancy nature. Multispectral image compression is done by translating the image data to a more compact form for storage and transmission purpose in remote sensing applications, thereby decreasing the cost and the complexity of the coding and decoding circuit. There are two types of redundancy present in multispectral images: spatial and spectral redundancy. Spatial redundancy refers to the correlation among the neighboring pixels due to the patterning, or self similarity within an image. The redundancy occurred due to the correlation between different color planes or spectral bands is called spectral redundancy. The objective of the compression technique is to reduce these spatial and spectral redundancies without any loss of image information to a maximum extent. To reduce these redundancies there are two types of compression techniques called as lossless image compression and lossy image compression. The reconstructed image from the compressed image is identical to the original image in case of lossless image compression which keeps the redundant information intact. Wherein a high compression ratio can be achieved with lossy image compression for which the reconstructed image is not identical to the original image.

\section{Proposed Compression Technique}

\subsection{Image Super-Resolution}

A hi-resolution (HR) image is obtained from a low-resolution (LR) input using Superresolution algorithm. There are mainly three methodologies for this approach: interpolation based methods, reconstruction based methods, and learning based methods. 
The simplest methods are interpolation methods but they tend to blur the high frequency details. Interpolation is the process of defining a function which estimates the values of a continuous function from discrete samples on specified values at specified points. Interpolation is used in Image processing applications like image magnification or reduction, by sub pixel image registration, to correct spatial distortions. By performing uniform sampling at or above the Nyquist rate a perfect reconstruction of image can be done using interpolation. The super resolution process is characterized using high frequency content of the images for interpolation technique. Properties of DWT make it interpolable [8]. Symlet wavelet functions are used to calculate DWT of the image. DWT gives four sub bands; three of these four sub bands contain high frequency contents of an image. High frequency sub band images (LH, HL and $\mathrm{HH}$ ) are enlarged by a factor of 2 using bicubic interpolation. LL sub band of wavelet domain is low pass filtered part of high frequency sub band. This means original image is rich in information compared to that of LL component. Instead of using LL component for getting inverse discrete wavelet transform we have used original image. This gives more detail information as compared to that of IDWT with LL component. Detailed flow chart of the image resolution enhancement with proposed method is shown in figure 1 . As shown in figure 1 high frequency sub bands of DWT are interpolated to get sub bands of same size as that of image. Input image and three components are used as input to IDWT which gives super resolution image.

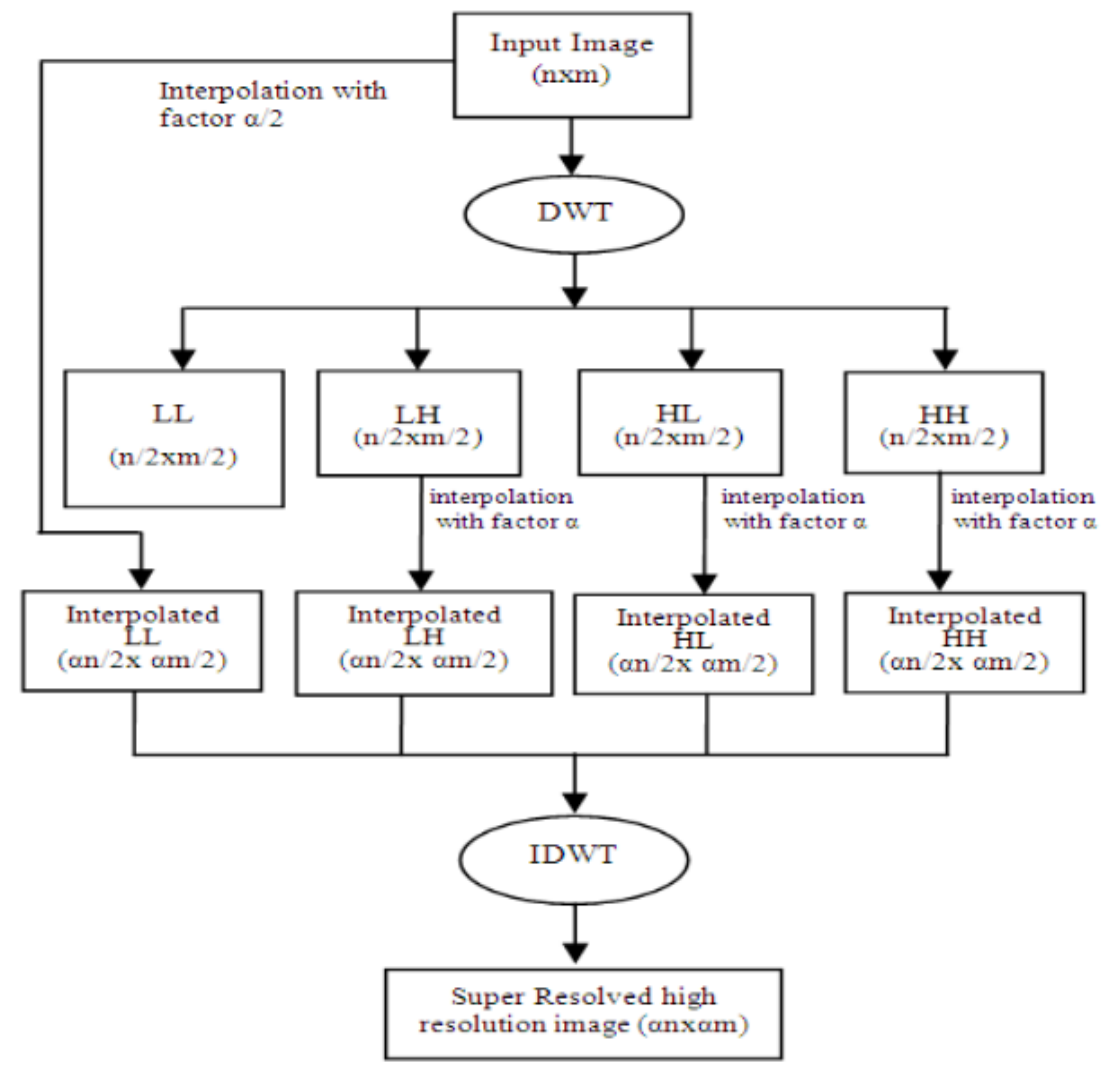

Figure 1. Image Enhancement using Interpolation Method

\subsection{Multi Resolution 2-D wavelet Decomposition}

In the proposed method, the maximum entropy and de-correlated bands are selected for the exploitation of spectral correlation that exists between the adjacent bands which contain maximum information. Two dimensional Discrete Wavelet Transform is applied on these representative bands which are then quantized and encoded using Improved 
SPIHT [9] algorithm. The spectral correlation which exists in wavelet coefficients of multispectral image are assumed for encoding process. This proposed scheme exploits the biorthogonal subclasses of the spatial and spectral correlation of the multispectral images. The proposed methodology consists of mainly two steps. In the first step, all bands of multispectral image are analyzed independently using two-dimensional symlet 8 DWT. In the second step the wavelet coefficients of maximum entropy and de-correlated bands are quantized and encoded using Improved SPHIT algorithm. ISPIHT is the wavelet based image compression method which provides the highest image quality [10].

3.2.1. Sub band Coding: The multispectral image is decomposed into band limited components, called sub bands, to reconstruct the original image without error using reassembled sub band coding. Since the bandwidth of the resulting sub bands $\mathrm{y}_{0}(\mathrm{n})$ or $\mathrm{y}_{1}$ (n) is smaller than the original signal $x(n)$, the sub bands can be down sampled without any degradation of information. The reconstruction of the original signal is obtained by up sampling, filtering, and summing the individual sub bands. In sub band coding, an image Figure 2 shows the components of two-band sub band coding and decoding system.

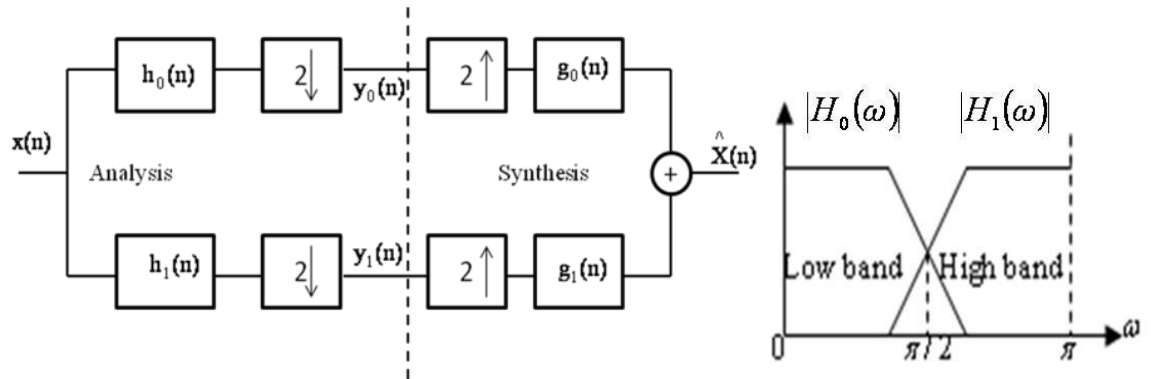

\section{Figure 2. Two-Band Filter Bank for One-Dimension Sub Band Coding and Decoding}

3.2.2. Multi Resolution Analysis: A scaling function is used in multi resolution analysis to create a series of approximations of a signal. A wavelet function is used to obtain the difference in information using encoding the data between adjacent approximations. A signal $\mathrm{f}(\mathrm{x})$ can be represented as a linear combination of expansion functions

$$
f(x)=\sum_{k} \alpha_{k} \phi_{k}(x)
$$

Where the $\alpha_{\mathrm{k}}$ is real-valued expansion coefficients and the $\varnothing_{\mathrm{k}}(\mathrm{x})$ are real-valued expansion functions and if this expansion is unique, the $\emptyset_{k}(x)^{\phi_{k}(x)}$ are called basis functions. The function space of the expansion set $\left(\emptyset_{\mathrm{k}}(\mathrm{x})\right)$ is

$V=\underset{k}{\operatorname{span}}\left\{\phi_{k}(x)\right\}$

And $f(x) \in V$ means that $f(x)$ is in the span of $\left(\varnothing_{k}(x)\right)$ and can be written in the form of Eq. (1). The coefficients $\alpha_{\mathrm{k}}$ are computed by taking the integral inner products of the dual $\tilde{\phi}_{k}(x)$ 's and function $\mathrm{f}(\mathrm{x})$. That is

$$
\alpha_{k}=\left\langle\tilde{\phi}_{k}(x), f(x)\right\rangle=\int \tilde{\phi}_{k}^{*}(x) f(x) d x
$$

If $\left(\emptyset_{\mathrm{j}, \mathrm{k}}(\mathrm{x})\right)$ is an orthonormal basis for $\mathrm{V}$, then $\phi_{k}(x)=\tilde{\phi}_{k}(x)$. If $\left(\emptyset_{\mathrm{k}}(\mathrm{x})\right)$ are not orthonormal but is an orthogonal basis function for $\mathrm{V}$, and the basis functions and their residual duals are called biorthogonal. That is 


$$
\left\langle\phi_{j}(x), \tilde{\phi}_{k}(x)\right\rangle=\delta_{j k}= \begin{cases}0 & , j \neq k \\ 1 & , j=k\end{cases}
$$

Now consider the set of expansion functions $\left(\varnothing_{\mathrm{j}, \mathrm{k}}(\mathrm{x})\right)$ composed of integer translations and its binary scales for the real, square-integral function $\emptyset(\mathrm{x})$ where

$\phi_{j, k}(x)=2^{j / 2} \phi\left(2^{j} x-k\right)$

for $k \in Z$ and $\varnothing(x) \in L^{2}(R)$. Because the shape of $\emptyset_{j, k}(x)$ changes with $j, \varnothing(x)$ is called a scaling function. The subspace spanned over $k$ is denoted for any $j$ as

$V_{j}=\operatorname{span}_{k}\left\{\phi_{j, k}(x)\right\}$

The scaling wavelet function has four prominent requirements of multi resolution analysis:

1. The integer translates are orthogonal for its scaling functions.

2. The subspaces which are spanned at low scales are nested within those spanned at higher scales by the scaling function. That is

$V_{-\infty} \subset \cdots \subset V_{-1} \subset V_{0} \subset V_{1} \subset V_{2} \subset \cdots \subset V_{\infty}$.

The only function that is common to all $V_{j}$ is $f(x)=0$.

That is $V_{-\infty}=\{0\}$.

Any function can be approximated with arbitrary precision.

That is, $V_{\infty}=\left\{L^{2}(\mathbf{R})\right\}$

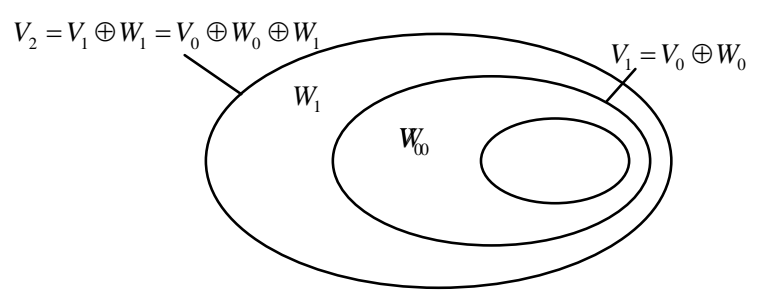

Figure 3. The Relationship between Scaling and Wavelet Function Spaces

$V_{j+1}=V_{j} \oplus W_{j}$

$L^{2}(\mathbf{R})=V_{0} \oplus W_{0} \oplus W_{1} \oplus W_{2} \oplus \cdots$

Or Even $L^{2}(\mathbf{R})=\cdots \oplus W_{-2} \oplus W_{-1} \oplus W_{0} \oplus W_{1} \oplus W_{2} \oplus \cdots$

3.2.3. Discrete Wavelet Transform: The wavelet series expansion function is denoted by the function $\mathrm{f}(\mathrm{x}) \in \mathrm{L}^{2}(\mathrm{R})$ relative to wavelet $\psi(\mathrm{x})$ and scaling function $\varnothing(\mathrm{x})$. We can write

$f(x)=\sum_{k} c_{j_{0}}(k) \phi_{j_{0}, k}(x)+\sum_{j=j_{0}}^{\infty} \sum_{k} d_{j}(k) \psi_{j, k}(x)$ 
Where $\mathrm{j}_{0}$ is an arbitrary starting scale and the $\mathrm{c}_{\mathrm{jo}}(\mathrm{k})$ are normally called the approximation or scaling coefficients, the $d_{j}(k)$ are called the detail of information or wavelet coefficients. The expansion coefficients are calculated as

$$
\begin{aligned}
& c_{j_{0}}(k)=\left\langle f(x), \tilde{\phi}_{j_{0}, k}(x)\right\rangle=\int f(x) \tilde{\phi}_{j_{0}, k}(x) d x \\
& d_{j}(k)=\left\langle f(x), \tilde{\psi}_{j, k}(x)\right\rangle=\int f(x) \tilde{\psi}_{j, k}(x) d x
\end{aligned}
$$

Sequences of numbers are used to expand the function, like samples of a continuous function $\mathrm{f}(\mathrm{x})$. Discrete wavelet transform (DWT) of $\mathrm{f}(\mathrm{x})$ is obtained by the resulting coefficients. Then the series expansion defined in Eqs. (14) and (15) becomes the DWT transform pair

$$
\begin{aligned}
& W_{\phi}\left(j_{0}, k\right)=\frac{1}{\sqrt{M}} \sum_{x=0}^{M-1} f(x) \tilde{\phi}_{j_{0}, k}(x) \\
& W_{\psi}(j, k)=\frac{1}{\sqrt{M}} \sum_{x=0}^{M-1} f(x) \tilde{\psi}_{j, k}(x) \\
& \text { for } j \geq j_{0} \text { and } \\
& f(x)=\frac{1}{\sqrt{M}} \sum_{k} W_{\phi}\left(j_{0}, k\right) \phi_{j 0, k}(x) \\
& +\frac{1}{\sqrt{M}} \sum_{j=j}^{\infty} \sum_{k} W_{\psi}(j, k) \psi_{j, k}(x)
\end{aligned}
$$

Where $f(x), \phi_{j_{0}, k}(x)$, and $\psi_{j, k}(x)$ are functions of discrete variable $\mathrm{x}=0,1,2, \ldots$, $\mathrm{M}-1$.

\subsubsection{Wavelet Transforms in Two Dimensions}

Two-dimensional scaling function, and three two-dimensional wavelet functions are required in a two dimensional wavelet transform,. Each is the product of a onedimensional scaling function $\phi$ and corresponding wavelet $\psi$.

$$
\begin{aligned}
& \phi(x, y)=\phi(x) \phi(y) \\
& \psi^{H}(x, y)=\psi(x) \phi(y) \\
& \psi^{V}(x, y)=\phi(y) \psi(x) \\
& \psi^{D}(x, y)=\psi(x) \psi(y)
\end{aligned}
$$

The variations along columns (like horizontal edges) are measured with $\psi^{\mathrm{H}}$, the variations along rows (like vertical edges) are measured with $\psi \mathrm{v}$, and the variations along diagonals are measured with $\psi \mathrm{D}$. The two-dimensional DWT can be implemented from one-dimensional discrete wavelet transform, using digital filters and down samplers. With the available separable two-dimensional scaling and wavelet functions, the onedimensional FWT of the rows of $\mathrm{f}(\mathrm{x}, \mathrm{y})$ is realized, followed by the one-dimensional FWT of the resulting columns. 


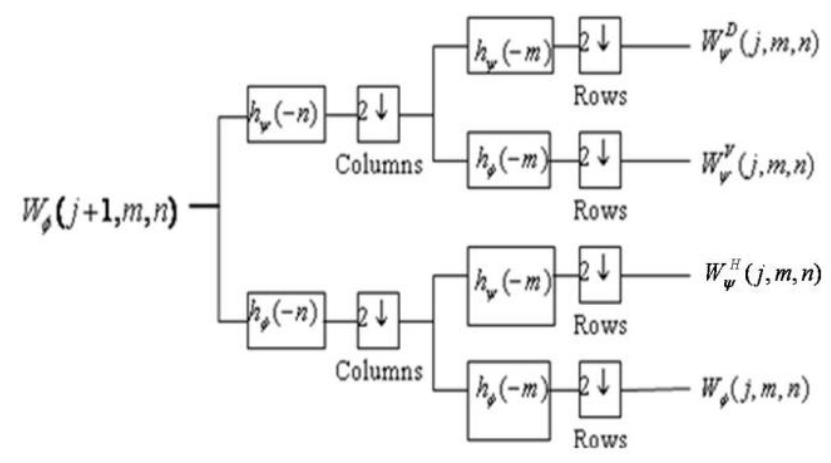

Figure 4. The Two-Dimensional FWT Analysis Filter Bank

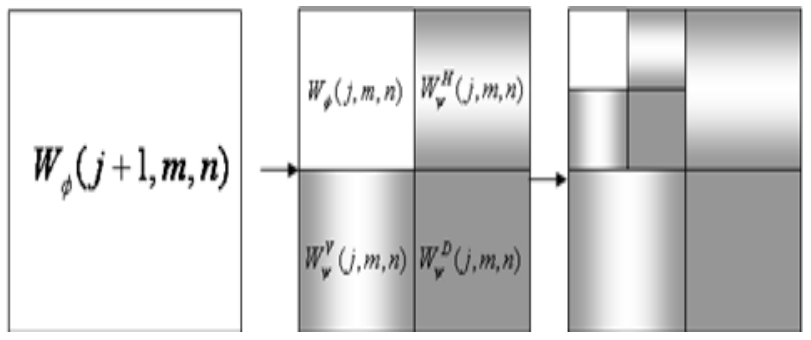

Figure 5. Two-scale of Two-Dimensional Decomposition

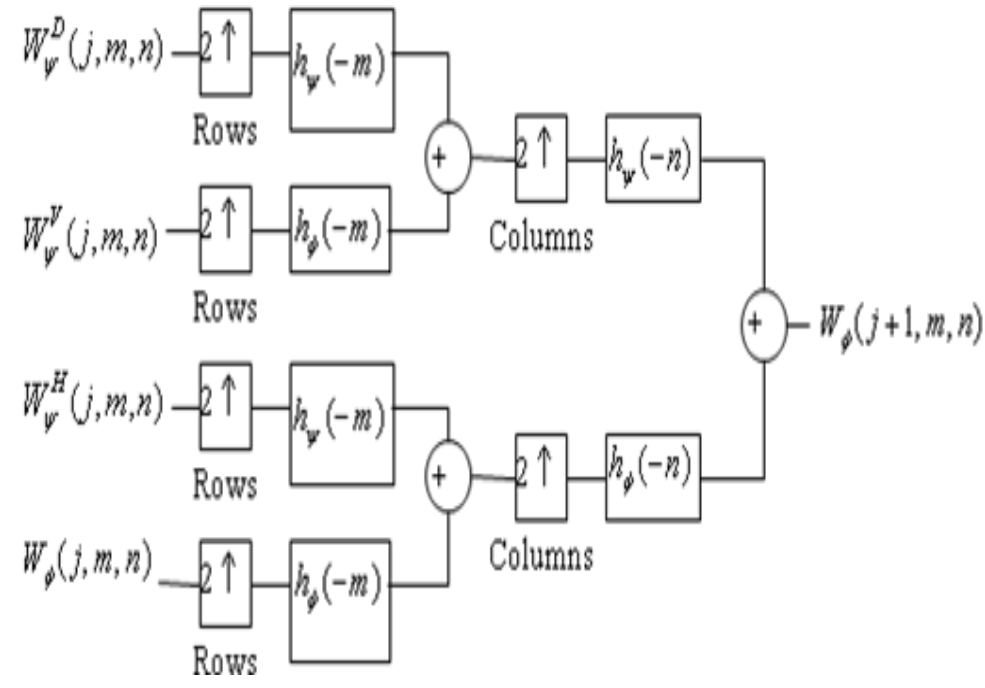

Figure 6. The Two-Dimensional DWT Synthesis Filter Bank

The single-scale filter bank of Figure 5 can be "iterated" by tying the approximation output to the input of another filter bank available to generate an arbitrary scaling transform. Like in the one-dimensional case, image $\mathrm{f}(\mathrm{x}, \mathrm{y})$ is used as the first scale input, and output four quarter-size sub images $-\mathrm{W}_{\varnothing}, \mathrm{W} \psi^{\mathrm{H}}, \mathrm{W} \psi^{\mathrm{V},}$ and $\mathrm{W} \psi^{\mathrm{D}}$. These sub images are shown in the middle of Figure 5. The available two iterations of the filtering process are required to produce the two-scale decomposition at the right of Fig 4. The synthesis filter bank which reverses the above process is described above in Figure 6.

\subsection{Improved Set Partitioning of Hierarchical Trees}

The Improved SPIHT algorithm is more efficient in terms of implementation when compared to the existing EZW (Embedded Zero Wavelet) [11] [12] algorithm which was 
presented by Shapiro. Wavelet transform image coding using traditional SPIHT has the advantages of embedded bit coding stream structure, with high compression rate, low complexity and easy to implement [13]. After applying wavelet transform to a multispectral image, the ISPIHT algorithm partions the decomposed wavelet into more significant and least insignificant partitions based on the following function:

$\operatorname{Sig}_{n}(T)=\left\{1, \max _{(\mathrm{i}, \mathrm{j}) \in T}\left|C_{i, j}\right| \geq 2^{n}\right.$

Here $\operatorname{Sig}_{n}(T)$ is the significance obtained from a set of coordinates $T$, and $c_{i, j}$ is the coefficient value at coordinate $(i, j)$. The maximum number of encoded bits required to represent the largest coefficient in the spatial orientation tree structure is achieved and represented by

$n_{\max }=\left[\log _{2}\left(\max _{(\mathrm{i}, \mathrm{j})}\left|C_{i, j}\right|\right)\right]$

There are mainly two passes in the algorithm- the sorting pass tree structure and the refinement pass tree structure. The ISPIHT encoding process utilizes three lists LIBT (List of Insignificant Block Test) - It contains individual coefficients as a block that have magnitudes smaller than the thresholds LIST (List of Insignificant Sets Test) - It contains set of wavelet coefficients that are described by the encoding tree structures and are found to have magnitudes lesser than the threshold. LSP (List of Significant Pixels) - It is a list of pixels found to have magnitudes greater than the threshold (significant) and $V T$ (vector Tree) corresponding to LIST. The sorting pass is performed on the above three lists. 


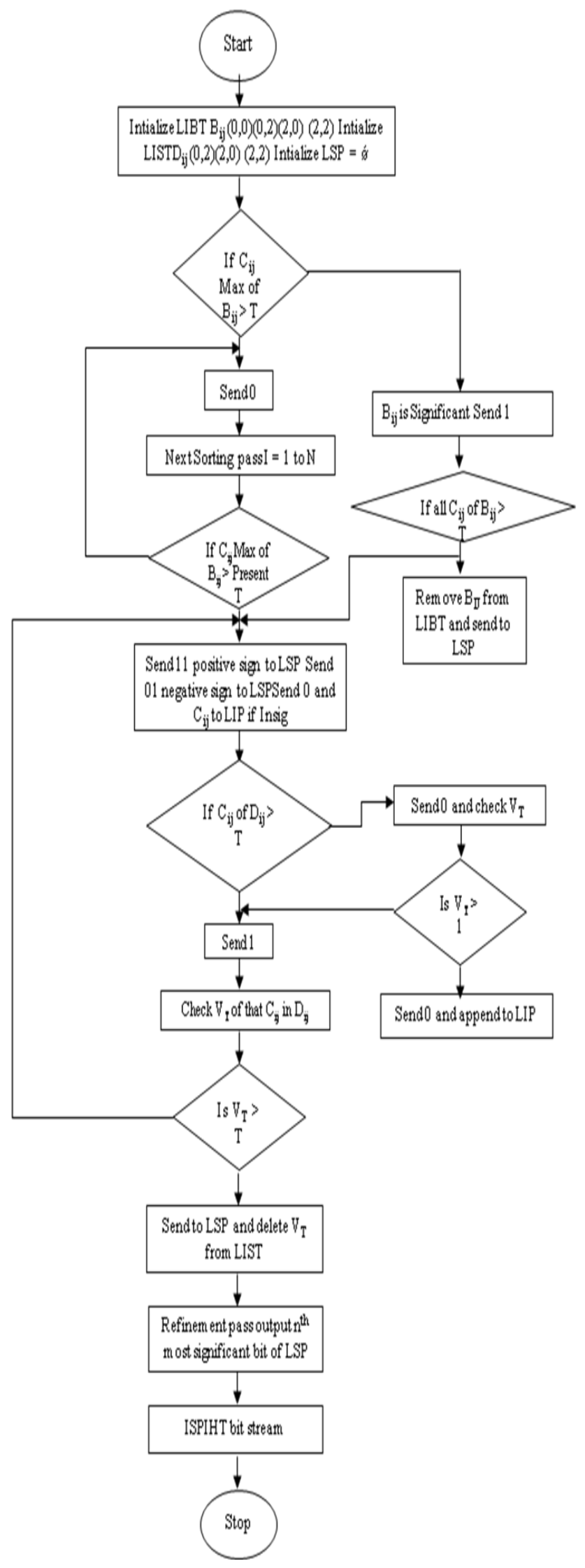

Figure 7. Flow Chart of Improved SPIHT Algorithm 


\section{Performance Evaluation}

Lossy compression performance traditionally is determined by simultaneously measuring the number of data operations which are required to perform both the encoding and decoding processes in terms of MSE, PSNR, CR, BER and SSIM.

\subsection{Mean Square Error (MSE)}

The most often used measurement is the mean square error (MSE). Mathematically, the mean squared error (MSE) is defined as.

$$
\mathrm{MSE}=\frac{1}{\mathrm{~N}_{1} \mathrm{~N}_{2}} \sum_{\mathrm{x}_{1} \mathrm{x}_{2}}\left(\mathrm{c}\left[\mathrm{x}_{1}, \mathrm{x}_{2}\right]-\mathrm{c}^{\prime}\left[\mathrm{x}_{1}, \mathrm{x}_{2}\right]\right)^{2}
$$

\subsection{Peak Signal-to-Noise Ratio(PSNR)}

$\operatorname{PSNR}=10 \log _{10}\left(\frac{2^{\mathrm{nsb}}-1}{\mathrm{MSE}}\right)$

Where nsb- maximum bit depth. MSE- means squared error between the original multispectral image and reconstructed multispectral image.

\subsection{Compression Ratio}

Compression ratio can then be determined as the ratio of the original dataset to the compressed data set.

$$
\mathrm{CR}=\frac{\mathrm{n}_{1}}{\mathrm{n}_{2}}=\frac{\text { Bits to store Original Image }}{\text { Bits to store Reconstructed Image }}
$$

\subsection{Structural Similarity Index Matrix}

The motivation behind the structural similarity approach is to measure the image quality with respect to human visual system (HVS) which is not designed for detecting imperfections or errors. Given any two images (of image patches) $\mathrm{x}$ and $\mathrm{y}$ which are to be compared, luminance is estimated as the mean of each image.

$\mu_{\mathrm{x}}=\frac{1}{\mathrm{~N}} \sum_{n=1}^{N} x_{n}$

Contrast is estimated using standard deviation as

$$
\sigma_{\mathrm{x}}=\sqrt{\frac{1}{\mathrm{~N}-1} \sum_{n=1}^{N}\left(x_{n}-\mu_{\mathrm{x}}\right)^{2}}
$$

and structure is estimated from the image vector $\mathrm{x}$ by removing the mean and normalizing by the standard deviation.

$$
\operatorname{SSIM}(x, y)=\left(\frac{2 \mu_{x} \mu_{\mathrm{y}}+\mathrm{C}_{1}}{\mu_{x}^{2}+\mu_{y}^{2}+\mathrm{C}_{1}}\right)\left(\frac{2 \sigma_{\mathrm{x}} \sigma_{\mathrm{y}}+\mathrm{C}_{2}}{\sigma_{x}^{2}+\sigma_{y}^{2}+\mathrm{C}_{2}}\right)\left(\frac{\sigma_{\mathrm{xy}}+\mathrm{C}_{3}}{\sigma_{\mathrm{x}} \sigma_{\mathrm{y}}+\mathrm{C}_{3}}\right)
$$




\section{Results and Discussions}

The Proposed algorithm was performed using MATLAB 2009b on an INTEL Pentium Core 2 duo (1.8 GHz, 2G RAM). We tested our algorithm on various multispectral image datasets like various bands of feathers (512X512) of cave multispectral image database which consists of 32 scenes, divided into 5 sections. Each scene also contains a single representative color image, which is displayed by using sRGB values rendered under a neutral daylight illuminant (D65).The input multispectral testing images of various bands of feathers of cave multispectral images are shown in Figure 8, and Figure 9. The image capture information is shown in Table 1. These multispectral images are used to represent the reflectances of the materials in the scene. The reflectance spectrum obtained at each pixel was computed from the measured multispectrum image using calibrated measured estimates of the illumination spectrum and camera spectral response. The matlab results for $0.8 \mathrm{bpp}$ for test band 5 of feathers is shown in Figure 13. The graphs for various testing band images of feathers for DCT KLT, DWT SPIHT and DWT ISPIHT compression methods for $0.8 \mathrm{bpp}$ are shown in the Figure10, Figure 11 and Figure12 respectively. The result analysis of various testing band images of feathers for DCT KLT, DWT SPIHT, and DWT ISPIHT for $0.8 \mathrm{bpp}$ are shown in Table 2, Table 3 and Table 4 respectively.
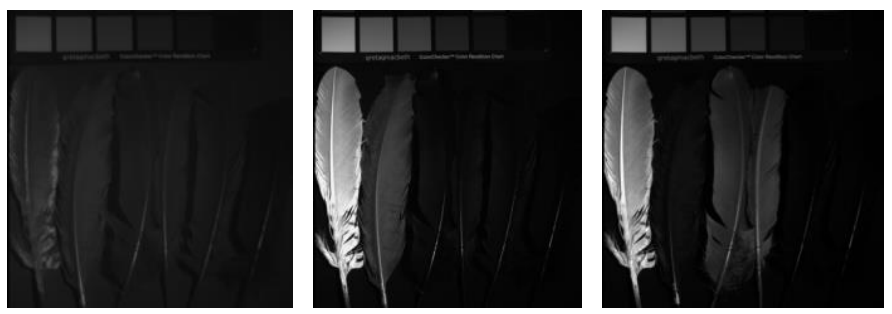

Figure 8. 512X512 Band 1, Band 5 and Band 11 Input Test Images of Multispectral Feathers Dataset
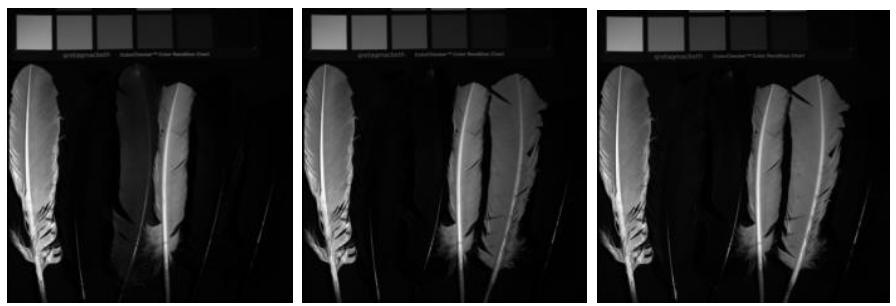

Figure 9. 512X512 Band 17, Band 23 and Band 27 Input Test Images of Multispectral Feathers Dataset

Table 1. Image Capture Information

\begin{tabular}{|l|l|}
\hline Camera & Cooled CCD camera (Apogee Alta U260) \\
\hline Resolution & 512 X512 pixel \\
\hline Filter & Vri Spec liquid crystal tunable filter \\
\hline Illuminant & CIE standard illuminator D65 \\
\hline Range of wavelength & 400nm $-700 \mathrm{~nm}$ \\
\hline
\end{tabular}




\begin{tabular}{|l|l|}
\hline Steps & $10 \mathrm{~nm}$ \\
\hline Number of band & 31 band \\
\hline Focal length & $\mathrm{f} / 1.4$ \\
\hline Focus & Fixed (focused using 550nm image) \\
\hline Image Format & PNG (16 bit) \\
\hline
\end{tabular}

Table 2. Result Analysis of DCT KLT Compression Method for 0.8bpp

\begin{tabular}{|c|c|c|c|c|c|}
\hline $\begin{array}{c}\text { Multispectral } \\
\text { Image Bands }\end{array}$ & PSNR & MSE & CR & BER & SSIM \\
\hline Band 1 & 21.681 & 441.538 & 0.170 & 1 & -0.011 \\
\hline Band 5 & 20.364 & 597.852 & 0.176 & 1 & -0.006 \\
\hline Band 11 & 20.290 & 608.216 & 0.187 & 1 & -0.007 \\
\hline Band 17 & 20.152 & 627.826 & 0.184 & 1 & -0.008 \\
\hline Band 23 & 20.122 & 632.145 & 0.177 & 1 & -0.006 \\
\hline Band 27 & 20.122 & 632.101 & 0.181 & 1 & -0.006 \\
\hline Band 31 & 20.716 & 551.365 & 0.184 & 1 & -0.011 \\
\hline
\end{tabular}

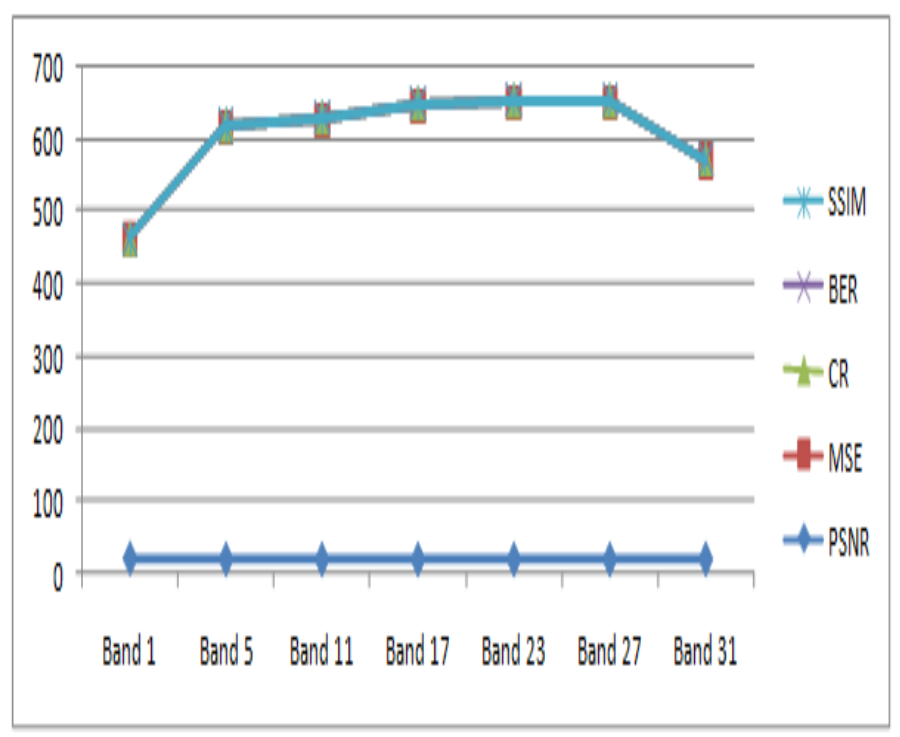

Figure 10. Graph for 0.8 bpp for DCT KLT Compression 
Table 3. Result Analysis of DWT SPIHT Compression Method for 0.8bpp

\begin{tabular}{|c|c|c|c|c|c|}
\hline $\begin{array}{c}\text { Multispectral } \\
\text { Image Bands }\end{array}$ & PSNR & MSE & CR & BER & SSIM \\
\hline Band 1 & 44.139 & 2.506 & 0.957 & 0.776 & 0.999 \\
\hline Band 5 & 43.271 & 3.061 & 0.983 & 0.789 & 0.971 \\
\hline Band 11 & 43.650 & 2.805 & 0.954 & 0.789 & 0.921 \\
\hline Band 17 & 43.484 & 2.915 & 0.976 & 0.791 & 0.891 \\
\hline Band 23 & 42.606 & 3.568 & 1.078 & 0.798 & 0.888 \\
\hline Band 27 & 42.274 & 3.851 & 1.133 & 0.799 & 0.920 \\
\hline Band 31 & 40.840 & 5.357 & 1.282 & 0.821 & 0.978 \\
\hline
\end{tabular}

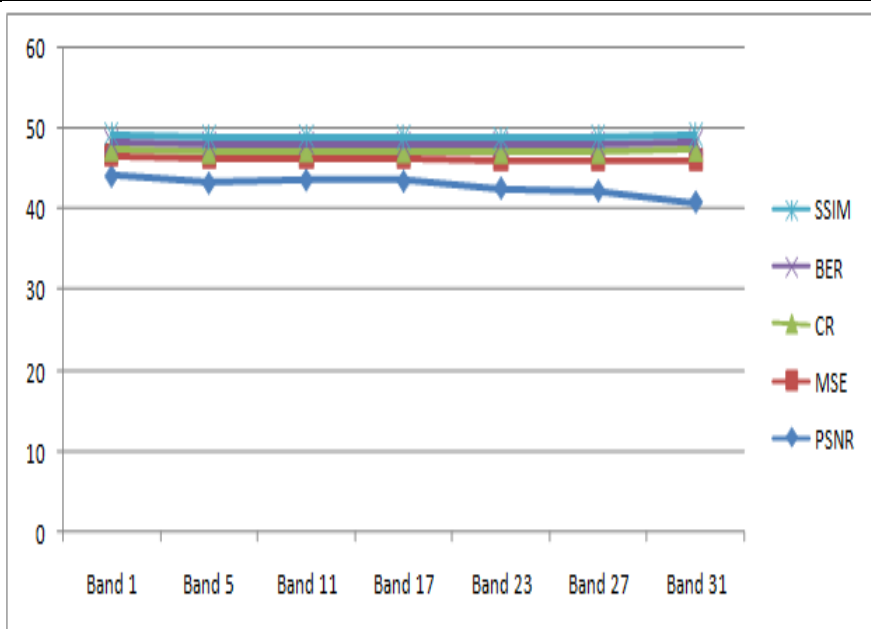

Figure 11. Graph for 0.8 bpp for DWT SPIHT Compression

Table 4. Result Analysis of DWT ISPIHT Compression Method for 0.8bpp

\begin{tabular}{|c|c|c|c|c|c|}
\hline $\begin{array}{c}\text { Multispectral } \\
\text { Image Bands }\end{array}$ & PSNR & MSE & CR & BER & SSIM \\
\hline Band 1 & 44.894 & 0.666 & 2.395 & 0.666 & 0.999 \\
\hline Band 5 & 53.832 & 0.269 & 1.999 & 0.269 & 0.971 \\
\hline Band 11 & 54.142 & 0.250 & 1.940 & 0.250 & 0.921 \\
\hline Band 17 & 53.731 & 0.275 & 2.175 & 0.275 & 0.891 \\
\hline Band 23 & 52.351 & 0.378 & 2.331 & 0.378 & 0.888 \\
\hline Band 27 & 52.240 & 0.388 & 2.5 & 0.388 & 0.920 \\
\hline Band 31 & 51.149 & 0.499 & 2.67 & 0.499 & 0.978 \\
\hline
\end{tabular}




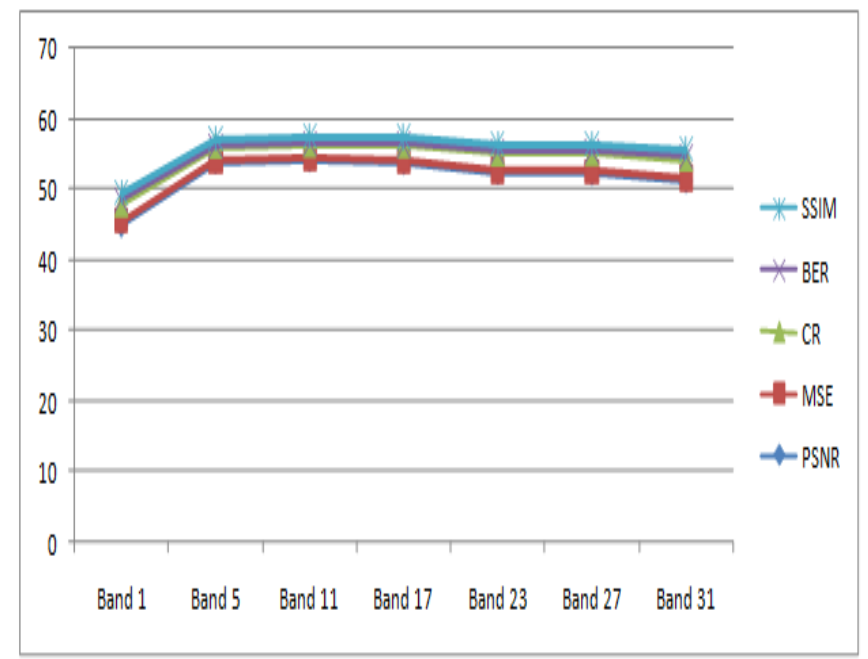

Figure 12. Graph for 0.8 bpp for DWT ISPIHT Compression

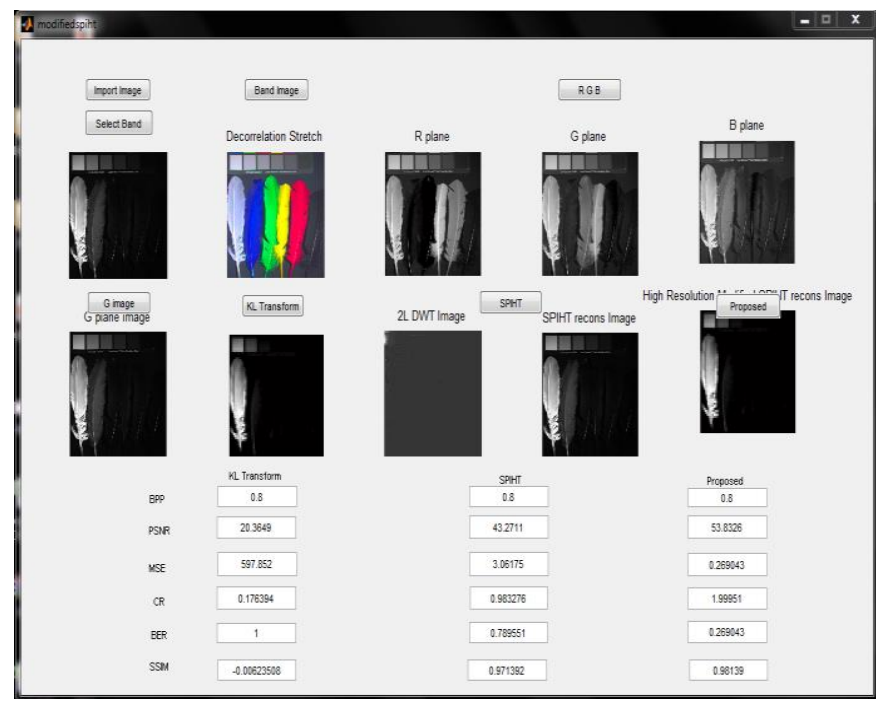

Figure 13. Matlab Results for Band 5 of Feathers Multispectral Image for 0.8 bpp

\section{Conclusion}

The proposed work consists of a new multi resolution encoding algorithm developed for multispectral image compression for various input multispectral image datasets. An interpolation super resolution image technique with 2D -Discrete wavelet transforms is performed on the input images. Quantization and encoding of the discrete wavelet coefficients using Improved SPIHT algorithm is performed for optimal bit-allocation, by assuming that there exists spectral correlation in the biorthogonal subclasses. DWT responds differently to bands of multispectral data and the bands which are similar in wavelet domain are encoded extremely well, which is confirmed by the results obtained by the proposed method when compared to the existing standard image compression techniques like DCT KLT, DWT SPIHT. Future work aims at extending this frame work for hyper spectral images and compressive multispectral imaging. 


\section{References}

[1] M. J. Weinberger, G. Seroussi and G. Sapiro, "The LOCO-I lossless image compression algorithm: Principles and standardization into JPEG-LS”, IEEE Trans. Image Processing, vol. 12, (2000) Augest, pp. 1309-1324.

[2] F. Rizzo, B. Carpentieri, G. Amott and J. A. Storer, "Low-Complexity Lossless Compression of Hyperspectral Imagery via Linear Prediction”, IEEE Signal Processing Letters, vol. 12, (2005) February.

[3] W. Zheng and H. Mingyi, "Multispectral imagery compression by hybrid DWT and partitioning DPCM", Journal of Electronics and Information Technology, vol. 125, (2003) Jun.

[4] X. Tang and W. A. Pearlman, "Hyperspectral Data Compression Three-Dimensional Wavelet -Based Compression", Chapter in Hyperspectral Images, Kluwer Academic Publishers, (2005).

[5] E. Magli, G. Olmo and E. Quacchio, "Optimized onboard lossless and near-lossless compression of hyperspectral data using CALIC, "IEEE Geosci. Remote Sens. Lett., vol. 1, (2004) January, pp. 21-25.

[6] M. J. Ryan and J.F. Arnold, "The lossless compression of AVIRIS images by vector quantization," IEEE Trans. Geoscience and Remote Sensing, Vol. 35, May 1997.

[7] Ian B and Joan S S 2010 IEEE Trans. Geosci.Remote Sens. 4872854.

[8] Fowler,J.E., The Redundant DiscreteWavelet Transform and Additive Noise. Signal Processing Letters, IEEE, 2005. 12(9): p. 629-632.

[9] A. Said,and W. A. Pearlman, " A new, fast and efficient image code based on set partitioning in hierarchical trees", IEEE Transactions on Circuits and Systems for Video technology, 1996, (6):243250.

[10] Yumnam Kirani Singh "ISPIHT-Improved SPIHT "A simplified and efficient subband coding scheme" Center for Development of Advanced Computing Plot: E-2/1, Block GP, Sector V, Salt Lake Electronics Complex.

[11] K.Sayood, "Introduction to Data Compression", 2nd edition, Academic Press, Morgan Kaufman Publishers, 2000 .

[12] Yumnam Kirani Singh "ISPIHT-Improved SPIHT "A simplified and efficient subband coding scheme" for Development of Advanced Computing Plot: E-2/1, Block GP, Sector V, Salt Center Lake Electronics Complex.

[13] F. W. Wheeler, and W. A. Pearlman, "SPIHT Image Compression without Lists," IEEE Int. Conf on Acoustics, Speech and Signal Processing (ICASSP 2000). Istanbul: IEEE: 2000.2047-2050.

\section{Authors}

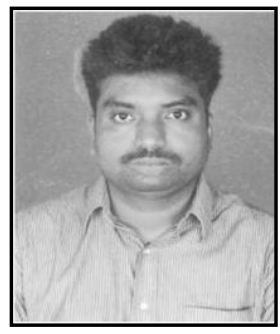

Prof. V.BhagyaRaju Completed his B.E. in 2000 from Vasavi college of engineering and M.Tech in Wireless \& Mobile Communications from JNTU-H. Presently he is pursuing Ph.D.from JNTU, Hyderabad in the field of Compression of Multispectral Images and working as a Professor in Department of ECE in Gurunanak Institutions Technical Campus,Hyderabad. He guided many research projects in M.Tech in the field of Image processing and VLSI.

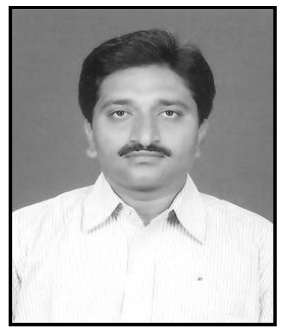

Dr. K Jaya Sankar received B.Tech degree in Electronics and Communication Engineering from NBKR Institute of Science and Technology, Vidya Nagar, and M.E \& Ph.D. degrees from the Department of ECE, OU, Hyderabad, Andhra Pradesh in 1988, 1994, and 2004 respectively. He is working as Principal at Vasavi College of Engineering, Hyderabad, India. He has 24 years of teaching experience in Digital Communication, Signal Processing, Electromagnetics, Antennas, Microwave and Radar Systems. His research areas of interest are in Coding Theory, RF \& Wireless Communications and Genetic Algorithm based antenna design. He has published more than twelve papers in national and international journals and more than twenty papers in national and international conferences. He is a Member of IEEE, Fellow of IETE and IE (I). 


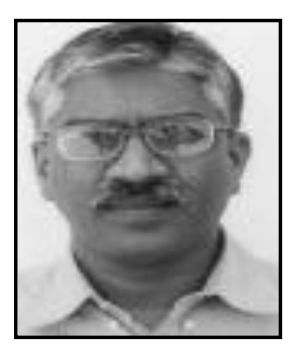

Dr. C. D. Naidu, an able administrator and accomplished educationalist, joined VNRVJIET in the year 1997 and has worked in the capacity of Vice-Principal, Dean-Academics, Professor \& Head-ECE Department and is at present Principal of the college. He was formerly associated with Vasavi College of Engineering, Hyderabad and KSRM College of Engg.Kadapa, A. P. His research areas include Digital signal processing, Digital filters, Neural Networks, Wavelet Transforms and Image Processing and Analysis. He has published and presented several papers in International and National Conferences and Journals. He has done his Ph.D in ECE from JNTU, Hyderabad, M.Tech in Instrumentation \& Control systems from S.V.University and B.Tech in ECE from JNTU.

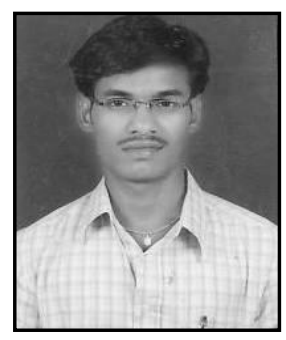

Srinivas Bachu, Research Scholar from GITAM University Hyderabad and M.Tech from JNT University, Hyderabad, Telangana. He has 9.8 years of teaching experience. At present B. Srinivas working with the Guru Nanak Institutions Technical Campus, Telangana, as an Assistant Professor in the Dept. of ECE. $\mathrm{He}$ is the Life Member of ISTE, AMIE, and IAENG He published two Text Books and 10 research papers at reputed International Conferences \& Journals. He is one of the Reviewer Board Member in four International Journals. His areas of interest are Signal, Image and Video Processing. 